\title{
AUTISMO: CONCEPÇÕES DE PROFISSIONAIS DE DIFERENTES ÁREAS SOBRE A INTERVENÇÃO PRECOCE
}

\author{
Autism: conceptions of professionals from different areas on early \\ intervention
}

Gabrielly Alberti ${ }^{1}$; Denise Aparecida Martins Sponchiado²

${ }^{1}$ Acadêmica do Curso de Pedagogia da Universidade Regional Integrada do Alto Uruguai e das Missões - Campus Erechim.E-mail: gabriellyalberti@hotmail.com

${ }^{2}$ Mestre em Educação. Professora Titular da Universidade Regional Integrada do Alto Uruguai e das Missões - Campus Erechim.E-mail: denisesponchiado@gmail.com

Data do recebimento: 18/08/2020 - Data do aceite: 14/10/2020

RESUMO: O artigo é recorte de um trabalho de conclusão do curso de graduação em Pedagogia, intitulado como "Autismo: Concepções de profissionais de diferentes áreas sobre a intervenção precoce" o qual traz a importância da intervenção precoce no transtorno do espectro autista (TEA). Teve como objetivo conhecer as percepções dos profissionais de diferentes áreas quanto à intervenção precoce em crianças diagnosticadas com TEA, a qual envolve uma grande variedade de serviços e de atividades em nível social, médico e pedagógico. É uma pesquisa de campo e a metodologia utilizada para realização da pesquisa foi qualitativa, centrando-se na compreensão e explicação dos oito profissionais entrevistados sobre o atendimento precoce de crianças autistas. Através da análise dos dados obtidos, nota-se que a maioria dos pacientes com TEA, que foram diagnosticados e tratados precocemente, podem evoluir e ter uma vida autônoma e funcional sem apresentar mais sintomas, o que é mais conhecido como "sair do espectro". A intervenção precoce deverá proporcionar "redes de apoio" formal e informal, coordenadas, com a finalidade de melhorar o bem-estar da unidade familiar, nuclear e alargada, promovendo o desenvolvimento da criança, respondendo assim às preocupações e necessidades da família através de um processo de capacitação e fortalecimento progressivo com vista à sua melhor adaptação e inserção social. Palavras-chave: Transtorno do Espectro Autista. Concepções. Intervenção Precoce. Inclusão. 


\begin{abstract}
This article is an excerpt from a final paper of an undergraduate course in Pedagogy entitled "Autism: Conceptions of professionals from different areas about early intervention" which brings the importance of early intervention in autism spectrum disorder (ASD). The aim of this study was to get to know the perceptions of professionals from different areas regarding the early intervention in children diagnosed with ASD, which involves a wide variety of services and activities at the social, medical and pedagogical level. It is a field research, and the methodology used was qualitative, focusing on the understanding and explanation of the eight professionals interviewed on the early care of autistic children. Through the analysis of the data obtained, it is noticed that the majority of the patients with ASD who were diagnosed and treated early can evolve and lead an autonomous and functional life without showing more symptoms, which is better known as "going off the spectrum". Early intervention should provide coordinated formal and informal "support networks", with the aim of improving the well-being of the nuclear and extended family, promoting the child's development, thus responding to the family's concerns and needs, through a qualification process and progressive strengthening with a view to better adaptation and social insertion.
\end{abstract}

Keywords: Autistic Spectrum Disorder. Conceptions. Early intervention. Inclusion.

\section{Introdução}

O Transtorno do Espectro Autista (TEA) compartilha sintomas centrais no comprometimento de três áreas específicas do desenvolvimento: déficits de habilidades sociais, déficits de habilidades comunicativas e presença de comportamentos repetitivos e estereotipados. As manifestações clínicas do TEA ocorrem antes dos 36 meses de idade e são mais percebidas especialmente quando as crianças são incluídas no contexto social. Segundo o Center for Disease Control, (BOSA; TEIXEIRA, 2017) a prevalência do TEA é de $1 / 68$, ou seja, pode atingir $1,47 \%$ dos indivíduos da população mundial. Observou-se prevalência nas últimas décadas em relação à intervenção precoce e ao diagnóstico do Transtorno em nosso país, porém, não houve relatos de como estas famílias estão sendo acolhidas após o diagnóstico.
Pode-se então definir a intervenção precoce: segundo Dunst e Bruder (2002, p. 68) como:

[...] o conjunto de serviços, apoios e recursos que são necessários para responder, quer as necessidades específicas de cada criança, quer as necessidades das suas famílias no que respeita à promoção do desenvolvimento da criança. Assim, intervenção precoce inclui todo o tipo de atividades, oportunidades e procedimentos destinados a promover o desenvolvimento e aprendizagem da criança, assim como o conjunto de oportunidades para que as famílias possam promover esse mesmo desenvolvimento e aprendizagem.

O Transtorno do Espectro Autista, apesar ser estudado há muito tempo, é ainda um distúrbio no qual existem ainda inúmeras questões por investigar, precisamente por ser um campo demasiado abrangente, complexo 
e com uma multiplicidade de variações dentro do mesmo.

De encontro com a necessidade, no que se diz respeito ao atendimento das pessoas com TEA, a pesquisa teve como objetivo conhecer as percepções dos profissionais de diferentes áreas quanto à intervenção precoce com crianças diagnosticadas com TEA. A metodologia utilizada se deu mediante uma pesquisa bibliográfica e de campo. Este trabalho é composto por entrevistas direcionadas a oito profissionais que apresentam as suas concepções sobre a intervenção precoce no TEA.

Serão apresentados os dados obtidos na pesquisa de campo, sob perspectiva exploratória e descritiva, com discussões geradas a partir dos autores estudados e seguindo a proposta inicial da pesquisa de fazer levantamento e analisar as concepções dos profissionais sobre a intervenção precoce no TEA.

\section{Conceituação do transtorno do espectro autista: definição e epidemiologia}

Distúrbios do espectro do autismo são distúrbios neurobiológicos caracterizados por déficits persistentes na comunicação social e na interação social entre ambientes. São distúrbios que se desenvolvem na primeira infância e geralmente continuam por toda a vida. É um distúrbio neurofisiológico e a sua causa é desconhecida, porém, alguns pesquisadores atribuem-no a alterações bioquímicas, mas outros associam-no a distúrbios metabólicos hereditários, encefalites, meningites, rubéola contraída antes do nascimento ou até mesmo lesões cerebrais. Mas ainda existem bastante incertezas e dúvidas na relação do Autismo com estas doenças.

O Transtorno do Espectro Autista (TEA) tem seu início precoce, curso crônico e tem por característica um desvio no desenvol- vimento da sociabilidade e nos padrões de comportamentos alterados (American Psychiatric Association apud Austimo: Avaliação psicológica e neuropsicológica, 2017. p. 07). A apresentação clínica desses quadros tem várias alterações, impactando em maior ou menor grau nas diversas áreas do desenvolvimento, como comunicação, socialização, aprendizado e adaptação a atividades da vida diária. No caso da socialização, alguns indivíduos que apresentam TEA podem demonstrar dificuldades graves de relacionamento social, enquanto outros aceitam passivamente as interações sociais, porém, não as iniciam e possuem dificuldade em mantê-las de forma habitual (HATTIER; MATSON, 2012; KLIN, 2006 apud BOSA; TEIXEIRA, 2017, p. 07).

Ter compreensão da variação dos quadros clínicos é um dos principais desafios para se ter o diagnóstico, portanto, é de grande importância preparar os profissionais para estarem atentos em suas práticas clínicas diárias, principalmente por que o Transtorno do Espectro Autista tem taxas elevadas na população em geral (APA, 2014 apud BOSA; TEIXEIRA, 2017. p. 07).

No novo sistema de diagnóstico, os Transtornos do Espectro do Autismo são divididos por níveis de gravidade: o indivíduo de nível 1 precisa de algum apoio como habilidades sociais e de comunicação; o indivíduo de nível 2 precisa de apoio substancial para se envolver em interações ainda mais estreitas e limitadas com outras pessoas; já o indivíduo do nível 3 precisa de um apoio substancial para a comunicação básica e o envolvimento com os outros.

O Transtorno do Espectro Autista pode ser dividido em três tipos: A Síndrome de Asperger é um transtorno neurobiológico enquadrado da categoria de transtornos globais do desenvolvimento. Segundo Medina (2019), por um tempo, foi considerada uma 
condição distinta, porém próxima e bastante relacionada ao autismo. Já o Transtorno Autista ou Autismo Clássico é um transtorno do desenvolvimento neurológico e deve estar presente desde o nascimento ou começo da infância. Como último tipo, o Transtorno Invasivo do Desenvolvimento, as pessoas que atendem alguns dos critérios de transtorno autista ou síndrome de Asperger, mas não todos, podem ser diagnosticadas com transtorno do desenvolvimento invasivo.

\section{Resultados e discussão}

Posteriormente, em conformidade com estudos realizados por autores sobre o assunto, a pesquisa concentrou-se no objetivo de conhecer as percepções dos profissionais de diferentes áreas quanto à intervenção precoce com crianças diagnosticadas com TEA. O estudo caracterizou-se como uma pesquisa qualitativa, onde os participantes pertencem ao universo de profissionais de diferentes áreas que atendem crianças autistas, atingindo um total de 08 profissionais, sendo 2 psicólogos, 1 psicopedagoga, 1 Terapeuta Ocupacional, 1 neurologista, 2 fonoaudiólogas, 1 pediatra. O critério da escolha dos profissionais se deu por conveniência. Para chegarmos aos profissionais se fez uma pesquisa informal, a partir de contato via e-mail, verificando se todos atendiam crianças com TEA e adquirindo consentimento em participar da pesquisa.

Após aprovação da pesquisa pelo Comitê de Ética em Pesquisa, optou-se por realizar um questionário semiestruturado, com perguntas abertas. Diante desse novo momento que estamos vivendo, a Pandemia do Coronavírus (COVID-19), mudou-se a forma de interpelar os diferentes profissionais que participaram da pesquisa. Com isto, foram enviadas, por e-mail, as perguntas do questionário dos oito profissionais de diferentes áreas. Todos responderam as questões.
Diante das respostas foram levantado as recorrências, as quais foram baseadas nos fragmentos de fala dos profissionais de diferentes áreas. Possibilitou-nos nomear as unidades de análise, que assim se configuraram: 1- início das experiências dos profissionais; 2- primeiros sinais e sintomas percebidos; 3-intervenções reconhecidas e recomendadas pelos profissionais; 4- intervenções reconhecidas e recomendadas pelos profissionais; 5- fases de luto.

Os enunciados eleitos para análise, aparentemente, podem complementar-se, diferenciar-se ou até antagonizar-se, já que os sujeitos participantes detêm conhecimentos e caminhadas distintas. Aquilo que enunciaram precisa ter eco, precisa reproduzir-se com vistas a produzir efeitos de sentido na busca de entendimentos possíveis para o que estava no foco. Sabe-se que só se visualizam ações quando transformadas em palavras; para isso, as palavras, servem e revestem-se de significados.

\section{A intervenção precoce é decisiva na evolução do tratamento}

\footnotetext{
"Muitos pais não reconhecem os sintomas leves do autismo e uma pequena porcentagem não presta atenção suficiente nas características do filho para poder identificar alguma alteração. Ainda, diagnósticos como timidez, hiperatividade ou "preguiça” para falar podem atrasar o diagnóstico de TEA."
}

Trazemos um excerto de uma médica quando afirma as dificuldades dos pais em perceber os sintomas de TEA nos seus filhos. Diante da fala da profissional que nos fez pensar sobre a pesquisa que teve como objetivo conhecer as percepções dos profissionais de diferentes áreas quanto à intervenção precoce com crianças diagnosticada com TEA, pode- 
-se notar que os pais não percebem, muitas vezes, os sintomas, por negarem que seu filho tenha algo diferente das outras crianças.

Os dados da pesquisa foram obtidos por meio de entrevistas enviadas por e-mail aos diferentes profissionais já citados, e após submetidos a um processo de análise de conteúdo e análise descritiva.

Para dar consistência argumentativa à pesquisa realizada, sempre que oportuno, falas ou fragmentos de fala dos profissionais que participaram das entrevistas serão inseridos no texto. Com a finalidade de garantir o anonimato dos participantes, optei por denominá-los, ficticiamente, como: Profissional Fonoaudióloga A, Profissional Psicóloga B, Profissional Neurologista C, Profissional Pedagoga D, Profissional Terapeuta Ocupacional E, Profissional Psicóloga F, Profissional Fonoaudióloga G e Profissional Psicopedagoga $\mathrm{H}^{1}$.

\section{Início das experiências dos profissionais}

Antigamente, o autismo não era comentado e nem estudado como atualmente, devido à falta de interesse nesta área. Sabe-se que o Transtorno do Espectro Autista possui algumas características especificas, como a dificuldade ou incapacidade de relacionamento com outras pessoas, atraso na linguagem e comportamentos repetitivos e restritos. Com isto, para se referir ao comportamento dos autistas, usava-se o termo "vivem em seu próprio mundo". Percebe-se isto, no fragmento de fala dos profissionais: ${ }^{2}$

Profissional Fonoaudióloga A - Atualmente minha atuação/atendimento de pessoas com TEA destina-se somente ao público infantil na faixa etária de 1 a 10 anos. Dentro disso as ações são muito variadas, as crianças são avaliadas e cada uma recebe um plano especifico de tratamento de acordo com as dificuldades que se apresentam, desde crianças muito pequenas que ainda não conseguem usar a comunicação até crianças maiores que já se comunicam verbalmente, mas que ainda possuem dificuldades em outras áreas como a aprendizagem de modo geral.

Profissional Psicóloga B - A minha experiência com crianças com TEA iniciou antes de eu finalizar minha graduação, há 8 meses como assistente terapêutica de terapia ABA (Análise do Comportamento Aplicada). Há 1 ano eu já realizava este trabalho, com supervisão de uma profissional habilitada, mas era com uma criança com suspeita de TEA, não confirmada posteriormente. Antes disso, só tive experiências como docente de língua inglesa para turmas em que crianças com TEA estavam incluídas, em função da minha formação inicial no curso Normal (Magistério).

Profissional Neurologista C - Durante a residência fazia atendimentos no Hospital da Criança Santo Antônio da Santa Casa de POA, preceptora pela Dra. Liselotte Barea. Atualmente, além dos atendimentos de consultório, trabalho diagnosticando e tratando pacientes com TEA também na APAE.

Profissional Pedagoga D - A inclusão de alunos com Deficiências no meu trabalho pedagógico é algo que sempre aconteceu de forma natural, muito antes da legislação vigente de inclusão ser aprovada. Alunos com diagnóstico clínico de TEATranstorno do Espectro Autista, é mais recente, devido a agilidade dos profissionais clínicos em atender e diagnosticar. Minha maior experiência com alunos com TEA, é na Sala de Recursos Multifuncionais que iniciei meu trabalho em 2008, anteriormente em sala de aula havia trabalhado com outras deficiências. Atualmente trabalho com crianças de 3 anos já diagnosticadas, que frequentam a Educação Infantil em sala de aula regular e o AEE no contra turno. Esse trabalho é em conjunto e complementar. 
Profissional Psicóloga $\boldsymbol{F}$ - iniciei minha experiência ainda sendo estagiaria do curso de psicologia realizei o estágio de 20 horas por semana realizando atendimentos clínicos e grupos com pais e grupos com jovens adultos. Quando terminei o estágio entrei como voluntaria na aquarela continuando com os atendimentos $e$ também atendo no consultório particular.

Percebe-se que existem diversas razões para o atraso do diagnóstico, como por exemplo a gravidade de cada caso suspeito, pois o quadro clinico de crianças com TEA de alto funcionamento pode passar despercebido ou ter seu diagnóstico confundido com de outros problemas de saúde. Porém, a razão que mais se destaca no atraso do diagnóstico é o despreparo dos próprios profissionais. Com a Profissional Psicopedagoga H e Profissional Terapeuta Ocupacional E, percebe-se o mesmo caso de falta de conhecimentos teóricos e práticos:

\section{Primeiros sinais e sintomas percebidos}

Em relação à natureza dos primeiros sintomas observados pelos pais, o atraso no desenvolvimento da comunicação e da linguagem é o sintoma relatado com maior frequência. Diversas situações também são percebidas pelas escolas que observam o desenvolvimento atípico das crianças e aconselham os pais a procurarem ajuda, como por exemplo: ausência de contato visual, movimentos estereotipados, brincadeiras repetitivas, falta de orientação ao ser chamado pelo nome, dificuldade em interagir com o meio, adultos ou com outras crianças da mesma idade.

Dentro disso, percebeu-se também relatos de pais que foram alertados do problema e procuraram ajuda, e outros pais que consideraram "normal" e acabaram não levando em conta os apontamentos dos professores, o que acabou atrasando ainda mais o diagnóstico crianças. Como nota-se na fala do Profissional Neurologista C:

\begin{abstract}
Profissional Neurologista $\boldsymbol{C}-$ Tudo depende muito do contexto social em que a criança está inserida. Quando há outras crianças na convivência, os pais percebem mais cedo a dificuldade de interação do paciente e de integração nas brincadeiras. Quando o paciente não possui outras crianças em seu entorno, o atraso na fala pode ser o primeiro sinal de alerta para os pais, por volta dos 2 anos de idade no caso de pacientes não-verbais. No caso de pacientes verbais com pouco contato com outras crianças, muitas vezes são os professores, nos primeiros meses de aula que percebem alguns sinais sutis da possibilidade diagnóstica.
\end{abstract}

Profissional Psicóloga B - Os familiares geralmente relatam sinais como atrasos à fala, desenvolvimento motor, ou perdas de habilidades já adquiridas, bem como dificuldades comportamentais de relacionamentos.

\section{Profissional Psicóloga F - Quando a criança já tem mais de 2 anos na maioria das vezes é onde os sinais aparecem mais declarados, normalmente quem dá o pri- meiro sinal é a escola pela interação da criança com outras crianças.}

Apesar de os primeiros sintomas descritos na literatura estarem relacionados à comunicação social e esses serem de fato um dos principais marcadores para distinguir crianças com TEA daquelas com múltiplos atrasos, alguns estudos com bebês demonstraram que outros sinais não ligados à sociabilidade podem ser identificados ainda precocemente, como por exemplo: irritabilidade, falta ou excesso de responsividade, alterações no nível de atividade e déficits no desenvolvimento motor. Porém, é importante ressaltar que em 
boa parte das crianças com TEA, o diagnóstico pode não estar claro antes dos 3 anos.

\section{Diagnóstico}

Segundo Caminha e colaboradores (2016, p. 48), o TEA pode se manifestar já nos primeiros meses de vida ou se apresentar após período inicial de desenvolvimento aparentemente normal seguido por regressão do desenvolvimento, ou seja, autismo regressivo. Nos indivíduos muito jovens, ressalta que em boa parte das crianças com TEA, o diagnóstico pode não estar claro antes dos 3 anos, porém, ainda assim é possível identificar sinais compatíveis com essas condições, que, quando são identificados, justificam o início do atendimento que deverá ser mantido até que os sintomas e sinais suspeitos desapareçam ou, prosseguir com o tratamento se for o caso presente de um TEA. Dentro disso, o Profissional Psicóloga B e a Profissional Fonoaudióloga $\mathrm{G}$, destaca que:

Profissional Psicóloga B - Não penso que o mais importante seja a certeza do "diagnóstico final" das crianças, mas preocupo-me com aquelas que não recebem intervenção enquanto não são diagnosticadas. Geralmente os cuidadores só buscam tratamento para a criança quando há uma definição diagnóstica. Mas penso que não somente o diagnóstico tenha esta finalidade... Acredito que, mesmo que o profissional não tenha critérios suficientes para o diagnóstico de TEA, a responsabilidade não é somente fazer uma reavaliação depois de um tempo, mas já realizar um encaminhamento para intervenção precoce, para que não seja perdida a oportunidade de estimular a criança e desenvolve-la para superar seus atrasos... Sejam cognitivos, motores, emocionais ou de qualquer outra ordem.
Profissional Fonoaudióloga G - Quando o paciente chega com suspeita, são avaliados os traços apresentados e caso se confirme a suspeita rapidamente é encaminhado para a avaliação neurológica, mesmo antes da confirmação do diagnóstico são trabalhadas as habilidades defasadas e é montado um plano de tratamento baseado nas dificuldades inerentes a cada paciente.

Profissional Terapeuta Ocupacional EIndependente se há ou não diagnóstico, deve-se iniciar a estimulação precoce na criança, seja com atraso, sindromes, com ou sem diagnósticos.

Segundo Caminha e colaboradores (2016, p. 49), a intervenção precoce pode ocorrer logo após o nascimento ou em seguida do diagnóstico, considerando-se até o período da primeira infância e tem-se como objetivos garantir que as crianças com distúrbios ou atrasos no desenvolvimento tenham atendimento adequado de acordo com a demanda, possivelmente com diminuição dos danos já causados e aumento das chances de melhor prognóstico, além da ampliação da rede de fortalecimento e apoio aos familiares.

Segundo Correia (2011), a intervenção precoce no autismo resume-se em atuar sobre os sintomas iniciais da patologia, evitando que estes se tornem irreversíveis e mais difíceis de tratar. Além disso, Dawson e Zanoli (apud CORREIA, 2011), defendem que, como o Transtorno do Espectro do Autismo está associado a alterações ao nível cerebral, a intervenção precoce pode possibilitar a criança com autismo a ter uma atividade cerebral mais normal, visto que esta provoca transformações nas sinapses neuronais que ainda se encontram flexíveis devido à plasticidade cerebral que estas crianças ainda apresentam. Assim conclui a Profissional fonoaudióloga A, Profissional Pedagoga D e a Profissional psicopedagoga $\mathrm{H}$ : 
Profissional Psicopedagoga H - Iniciei quando chegou ao consultório uma criança com características de TEA. Como tinha pouco conhecimento encaminhei para outro profissional e fui à busca de conhecimento. Realizei cursos na Pandorga (instituição de formação em Autismo), Curso de neurologia com a Dra. Newra Rotta, e vários outros cursos para após conviver com profissionais que atuavam na área e realizar alguns atendimentos com supervisão, iniciar os atendimentos no consultório.

\section{Profissional Terapeuta Ocupacional E- $O$ autismo não era comentado e estuda- do. Era dito que eram pessoas que viviam em seu mundo particular, mas não havia manejos e ferramentas para acessar esse mundo. Sempre brinco que apanhei muito deles, por insistir em atividades e condutas! Pura falta de conhecimentos teóricos e práticos.}

\section{Intervenções reconhecidas e recomendadas pelos profissionais}

Alguns estudos afirmam que o planejamento do tratamento do TEA deve ser estruturado de acordo com as etapas de vida do paciente. Portanto, o trabalho com crianças pequenas deverá analisar como prioridade a terapia da fala, da interação social/linguagem, educação especial e também o suporte familiar. Já com pacientes adolescentes, o alvo deverá ser os grupos de habilidade social, terapia ocupacional e sexualidade. Com adultos, questões como independência, moradia e tutela deverão ser focadas. Alguns métodos foram citados pelos profissionais entrevistados:

\footnotetext{
Profissional Psicóloga B - Quando a
} criança recebe estimulação precoce, o cérebro dispõe de uma capacidade muito maior de conexões neurais frente a estímulos. Entendo que durante estas janelas de oportunidades, a criança tem chances muito maiores de uma aprendizagem sólida, de adquirir habilidades-chave e comportamentos que facilitarão o posterior desenvolvimento de habilidades progressivamente mais refinadas. A intervenção tem chances de trazer mais resultados quando realizada precocemente, além de prevenir o desenvolvimento de comportamentos indesejados, no caso da Análise do Comportamento Aplicada. A criança, portanto, terá mais chances de adaptação ao seu meio e poderá ter atrasos menos significativos.

Profissional Fonoaudióloga A - Em minha prática utilizo ABA, DIR - Floortime, TEACH, PECS. Acredito ser muito importante conhecer as estratégias de cada uma, porém, na prática a maioria dos profissionais não segue apenas uma única linha e sim incorpora conhecimentos obtidos delas de acordo com a necessidade de cada criança e cada família.

\section{Profissional Psicóloga $\boldsymbol{F}$ - A Terapia Cognitivo Comportamental ajuda mui- to para o tratamento juntamente com o $A B A$, Denver e outros, porém, isso varia muito cada caso. Cada autista é único e o plano terapêutico também.}

Nas entrevistas, foi chamado a atenção para a variedade de serviços disponíveis, desde aqueles com abordagens individuais e seguindo uma única linha, até aqueles compostos por clínicas multidisciplinares que incorporam conhecimentos obtidos de acordo com as necessidades de cada criança e cada família. Enfatizou-se que a eficácia do tratamento depende da experiência e do conhecimento dos profissionais sobre o autismo e, principalmente, de sua habilidade de trabalhar em equipe e com a família. Segundo Gaiato (2018, p. 85), a intervenção deve ser feita na clínica, na casa e na escola da criança. 
A estimulação ideal para o autismo é de 15 a 40 horas semanais, mas para isso ocorrer, os pais são orientados por profissionais especializados e dão continuidade aos estímulos em casa.

Das intervenções reconhecidas e recomendadas pelos profissionais questionados, temos:

1. Modelo Denver de Intervenção: É baseado integralmente na Análise Aplicada do Comportamento (ABA). Foi considerado pela revista Times, em 2012, uma das dez maiores descobertas da área médica. Trata-se de uma abordagem de intervenção com comprovação científica que otimiza o desenvolvimento das crianças com autismo na faixa entre um a cinco anos de idade. Este modelo tem como prioridade a construção das interações sociais da criança, a espontaneidade e habilidade de engajamento com o outro, o que leva a construção de vínculos de afeto de forma positiva e natural. O Modelo Denver tem como objetivo ajudar a criança a aprender em todas as situações do dia a dia, pois explora de forma ativa as oportunidades de aprendizagens. (GAIATO, 2018, p. 88).

2. Terapia ABA: ABA (Análise Aplicada do Comportamento) trata-se de uma ciência, com um conjunto de princípios que já foram amplamente pesquisados e comprovados como eficientes. Existem diversas habilidades que são trabalhadas durante todo o processo terapêutico: comportamentos sociais, comunicação funcional e contato visual, comportamentos acadêmicos que são requisitos para escrita, leitura, interpretação e matemática, além da redução de comportamentos como as estereotipias, autolesões e agressões. (GAIATO, 2018, p. 87).

3. TEACCH: É a sigla de Tratamento e Educação de Crianças Autistas e com desvantagens na Comunicação. Baseia sua metodologia no princípio de que todas as crianças com autismo podem aprender, mesmo que de formas diferentes. Utiliza apoios principalmente visuais para ensinar comportamentos. (GAIATO, 2018, p. 89).

4. PECS: Sistema de Comunicação por Troca de Figuras é um método para ensinar pessoas com distúrbios de comunicação e/ou com autismo a comunicarem-se de forma funcional por intermédio da troca de figuras. Estudos sobre a metodologia PECS mostram que esse método aumenta a intenção comunicativa das pessoas com autismo. (GAIATO, 2018, p. 89).

As características do TEA afetam condições físicas e mentais dos indivíduos, aumentando a demanda por cuidados e, consequentemente, o nível de dependência de pais e/ou cuidadores. Com isso, as famílias de indivíduos autistas, se veem frente ao desafio de ajustar seus planos e expectativas quanto ao futuro. $\mathrm{O}$ momento do diagnóstico de uma doença ou síndrome, para a família, é composto por um conjunto de sensações e sentimentos diversos: frustração, insegurança, culpa, medo, luto e desesperança, principalmente quando o paciente é uma criança.

Constata-se a partir das entrevistas que um dos motivos pelos quais os pais deixam de procurar ajuda profissional é a falta de conhecimento, apesar de já ter aumentado consideravelmente o acesso dos cuidadores às informações sobre o desenvolvimento e sinais de alerta para transtornos na infância.

\footnotetext{
Profissional Neurologista C - Muitos pais não reconhecem os sintomas leves do autismo e uma pequena porcentagem não presta atenção suficiente nas características do filho para poder identificar alguma alteração. Ainda, diagnósticos como timidez, hiperatividade ou "preguiça" para poder falar, podem sim atrasar o diagnóstico de TEA.
} 
Profissional Fonoaudióloga $\boldsymbol{G}$ - A falta de conhecimento faz a família ignorar a gravidade da postergação do início do tratamento. A negligência se dá por vezes pela não aceitação do quadro, onde a família necessita orientação psicológica para trabalhar o processo ao qual está passando. E no caso dos outros diagnósticos, na prática se vê que são muitos que opinam sobre o possivel diagnóstico (família, professores), atrasando uma avaliação adequada e fazendo com que a família adie a investigação do quadro.

\section{Fases do luto}

De acordo com alguns profissionais entrevistados, o impacto de um diagnóstico de uma doença pode propiciar à família a vivenciar as mesmas fases do luto, inclusive a negação, raiva e culpa. Sem dúvida, a presença de uma criança diagnosticada como deficiente em uma família cria uma situação, ou seja, um problema novo, nunca antes enfrentado por muitas famílias. Em geral, a chegada do bebê que apresenta uma deficiência torna-se um evento traumático e desestruturador, que interrompe o equilíbrio familiar.

Outro aspecto que causa impacto e compromete a aceitação da doença pela família, remete-se a demora na conclusão do diagnóstico clínico. Isso porque, enquanto não se tem um diagnóstico fechado, os pais têm esperanças de que o problema da criança é mais simples, o que pode criar falsas expectativas de que os sinais e sintomas apresentados é alto transitório.

Profissional Psicopedagoga $\boldsymbol{H}$ - Temos que respeitar as fases do luto pelos pais, principalmente os que atendem o público infantil precisam estar alertas e buscando conhecimentos atualizados sobre neurodesenvolvimento que possibilitem condutas adequadas nos casos suspeitos de TEA elou quaisquer outras alterações.
Profissional Psicóloga B - Acredito que outro motivo seria um processo emocional de negação pelo qual os pais podem passar com relação às dificuldades dos filhos. São muitas as expectativas parentais com relação ao desenvolvimento do filho e pode ser muito doloroso aceitar que os atrasos do seu filho são reais e significativos.

A forma como é transmitida a notícia pode influenciar fortemente nas reações vivenciadas pelos pais, sendo que muitas vezes o momento do diagnóstico é algo que se mostra confuso, podendo interferir na vinculação com essa criança e, também, podendo gerar falsas expectativas com relação a ela, influenciando assim na aceitação dela pela família. Pode-se perceber que a revelação diagnóstica do Transtorno do Espectro Autista ocasiona importantes repercussões no contexto familiar.

A expectativa do filho idealizado é frustrada devido ao desconhecimento acerca da síndrome, o que fortalece a concepção da necessidade de um melhor apoio, atenção e principalmente orientação por parte profissional que notificará a descoberta do autismo. Por estas, entre outras razões, vale salientar que é de suma importância o reconhecimento e a procura por tratamento o quanto antes, pois, o mesmo pode reduzir bastante os danos, proporcionado uma melhor qualidade de vida tanto para família quanto para o autista.

\section{Considerações finais}

O TEA, ou simplesmente autismo, é um transtorno de neurodesenvolvimento, os sintomas variam e estão presentes desde os primeiros anos de vida, ou até mesmo nos primeiros meses. A partir da pesquisa realizada, conclui-se que o número de profissionais que trabalham nesta área é baixo em comparação 
à grande demanda de casos que começaram a surgir atualmente.

Diversos profissionais relatam que, os sinais são percebidos na primeira infância pelos pais, principalmente entre os 18 e 36 meses de idade da criança, como: atrasos com relação à fala, desenvolvimento motor, ou perdas de habilidades já adquiridas, bem como dificuldades comportamentais e de relacionamento.

A grande maioria dos profissionais afirmam que mesmo com o diagnóstico clínico de TEA não fechado, a intervenção é iniciada de qualquer forma devido a sua grande importância em trabalhar a partir dos atrasos de desenvolvimentos que foram identificados no indivíduo. Com isso, deve ser ressaltado que quando se fala em intervenção precoce, sempre é importante pensar em uma ampla gama de profissionais que poderão estar atuando com a criança: fonoaudiólogos, psicopedagogos, terapeutas ocupacionais, fisioterapeutas e pedagogos.

Percebemos ainda que há muitos motivos para os pais/familiares não procurarem ajuda dos profissionais, sendo: falta de conhecimento, processo emocional de negação pelo qual os pais passam, críticas, preconceitos e a inviabilidade financeira. Diversas situações foram relatadas de que as escolas observam o desenvolvimento atípico das crianças e comunicam os pais a procurarem ajuda, mas os mesmos não acabam levando a sério e não vão em busca de explicações. Por outro lado, há pais que depois de alertados, vão em busca de ajuda profissional e acabam tendo o diagnóstico clínico fechado cedo, assim ajudando a criança a ter mais tempo para se desenvolver.

Com a pesquisa realizada, sabemos que os sintomas podem ser reduzidos por meio da compreensão e da conduta correta dos casos. Não existe um tratamento único, sendo assim, não existe uma cura para o autismo.

Com isso, conclui-se que o autismo é um transtorno importante, mesmo quando os sintomas são leves. Alguns profissionais acreditam que, dependendo de cada caso, a maioria dos pacientes com TEA podem evoluir a ter uma vida autônoma e funcional sem apresentar mais sintomas, o que é mais conhecido como "sair do espectro". O passo mais importante para o tratamento do autismo é aceitar e encarar o problema de frente, para assim, conseguir desenvolver o paciente para uma vida sem dependências.

Para finalizar cabe destacar que o tema não se encerra com a presente pesquisa, muito pelo contrário, demonstra que pode e deve ser continuada por quem gosta de estudar sobre a temática em questão.

\section{NOTAS}

${ }^{1}$ Os fragmentos de fala dos profissionais participantes serão marcados, no corpo do texto, apenas com uma aspa.

2 Os fragmentos de fala dos profissionais participantes da pesquisa serão transcritos em itálico, numa caixa de texto, fonte 10, times new roman, diferenciando-os, assim, de citações e de outros textos utilizados no trabalho. 


\section{REFERÊNCIAS}

BANDEIRA, D. R.; TRENTINI, C. M.; KRUG, J. S. Psicodiagnóstico - Psicodiagnóstico: formação, cuidados éticos, avaliação de demanda e estabelecimento de objetivos. Porto Alegre, Artmend, 2016.

BOSA, C. A.; TEIXEIRA, M. C. T. V. Autismo: Avaliação psicológica e neuropsicológica. 2. ed. São Paulo: Hogrefe, 2017.

BOSA, C.; SCHMIDT, C. A investigação do impacto do autismo na família: Revisão crítica da literatura e proposta de um novo modelo. 2003, IFRS. Disponível em: file://C:/Users/Compaq/ Downloads/3229-6359-1-PB.pdf. Acesso em: 20 jun. 2020.

CAMINHA, V. L. et al. Autismo: Vivências e Caminhos. 2016. São Paulo. Disponível em: http://pdf. blucher.com.br.s3-sa-east-1.amazonaws.com/openaccess/9788580391329/completo.pdf\#page=46. Acesso em: 14 jun. 2020.

CORREIA, N. C. C. C. A importância da intervenção precoce para as crianças com autismo na perspectiva dos educadores e professores de educação especial. 2011. Lisboa. Disponível em: http://recil.grupolusofona.pt/bitstream/handle/10437/1489/Neusa\%20Correia.pdf.pdf?sequence=1. Acesso em: 17 jun. 2020.

GAIATO, M. S.O.S - Autismo: Guia completo para entender o Transtorno Do Espectro Autista. São Paulo: N. Versos. 2018.

HENNES, J. L.; RODES, M. Associação Psiquiátrica Americana - Manual Diagnóstico e Estatístico de Transtornos Mentais. 2002. Washington. Disponível em: https://www.sciencedirect. com/science/article/pii/B978143772242000016X?via\%3Dihub. Acesso em: 10 jun. 2020.

HENNES, J. L.; RODES, M. Associação Psiquiátrica Americana - Manual Diagnóstico e Estatístico de Transtornos Mentais - DSM-V. 5. ed. 2014. Washington. Disponível em: https:// www.sciencedirect.com/science/article/pii/B978143772242000016X?via\%3Dihub. Acesso em: 10 jun. 2020.

LAMPREIA, C. Perspectiva da pesquisa prospectiva com bebês irmãos de autistas. Vol 29. 2009. Brasília. Disponível em: https://www.scielo.br/scielo.php?script=sci_arttext\&pid=S14149893200900 $0100013 \& \operatorname{lng}=$ pt\&tlng=pt. Acesso em: 12 jun. 2020.

MAENNER, M. J. et al. Centers for Disease Control and Prevention: Prevalência de Transtorno do Espectro do Autismo em Crianças de 8 Anos - Rede de Monitoramento do Autismo e Deficiências do Desenvolvimento. Estados Unidos, 2016. Disponível em: https:/www.cdc.gov/mmwr/ volumes/69/ss/ss6904a1.htm?s_cid=ss6904a1_w. Acesso em: 16 maio 2020.

MAIA, F. A. et al. Importância do acolhimento de pais que tiveram diagnóstico do transtorno do espectro do autismo de um filho. Rio de Janeiro, 2016. Disponível em: https://www.scielo.br/ pdf/cadsc/v24n2/1414-462X-cadsc-24-2-228.pdf. Acesso em: 16 maio 2020.

MEDINA, Y. Transtorno do Espectro do Autismo. Transtorno do espectro autista pode ser dividido em três tipos. Folha Noroeste, Frederico Westphalen. 15 fev.2019. Disponível em:https://www. folhadonoroeste.com.br/colunas/transtorno-do-espectro-do-autismo/\#: :text=O\%20transtorno $\% 20$ do $\% 20$ espectro\%20autista,e $\% 20$ bastante $\% 20$ relacionada $\% 20$ ao\%20autismo. Acesso em: 04 nov. 2020 
NAZARI, A. C. G.; NAZARI, J.; GOMES, M. A. Transtorno do espectro autista: discutindo o seu conceito e métodos de abordagem para o trabalho. Uberlândia, 2017. Disponível em: http://www. eventos.ufu.br/sites/eventos.ufu.br/files/documentos/transtorno_do_espectro_autista_discutindo_o_ seu_conceito_e_metodos_de_abordagem_para_o_trabalho.pdf. Acesso em: 18 maio 2020.

PIMENTEL, J. S. Avaliação de programas de intervenção precoce. Lisboa, 2004. Disponível em: file://C:/Users/Compaq/Downloads/128-354-1-PB.pdf. Acesso em: 18 maio 2020.

PINTO, R. N. M. et al. Autismo infantil: impacto do diagnóstico e repercussões nas relações familiares. Paraíba, 2016. Disponível em: https://www.scielo.br/pdf/rgenf/v37n3/0102-6933rgenf-1983-144720160361572.pdf. Acesso em: 20 jun. 2020.

SEIMETZ, G. D. Avaliação psicológica da criança com suspeita de transtorno do espectro autista: desafios para o avaliador. 2018. Porto Alegre. Disponível em: https://www.lume.ufrgs.br/ bitstream/handle/10183/193377/001091963.pdf?sequence=1. Acesso em: 12 jun. 2020.

TUCHMAN, R.; RAPIN, I. Autismo: Abordagem Neurobiológica. Porto Alegre, Artmed, 2009. ZANON, R. B.; BACKES, B.; BOSA, C. A. Identificação dos Primeiros Sintomas do Autismo pelos Pais, v. 30. 2014. IFRS. Disponível em: https://www.scielo.br/pdf/ptp/v30n1/04.pdf. Acesso em: 12 jun. 2020. 
